\title{
REPRESENTING COMPLEX ANALOGUES USING A FUNCTION MODEL TO SUPPORT CONCEPTUAL DESIGN
}

\author{
L Siddharth \\ Indian Institute of Science \\ Bengaluru, Karnataka, India
}

\author{
Amaresh Chakrabarti \\ Indian Institute of Science \\ Bengaluru, Karnataka, India
}

\author{
Srinivasan Venkataraman \\ Indian Institute of Technology Delhi \\ New Delhi, India
}

\begin{abstract}
Analogical design has been a long-standing approach to solve engineering design problems. However, it is still unclear as to how analogues should be presented to engineering design in order to maximize the utility of these. The utility is minimal when analogues are complex and belong to other domain (e.g., biology). Prior work includes the use of a function model called SAPPhIRE to represent over 800 biological and engineered systems. SAPPhIRE stands for the entities: States, $\underline{\text { Actions, }}$ Parts, Phenomena, Inputs, oㅁgans, and $\underline{\mathbf{E} f f e c t s}$ that together represent the functionality of a system at various levels of abstraction. In this paper, we combine instances of SAPPhIRE model for representing complex systems (also from the biological domain). We use an electric buzzer to illustrate and compare the efficacy of this model in explaining complex systems with that of a well-known model from literature. The use of multiple-instance SAPPhIRE model instances seems to provide a more comprehensive explanation of a complex system, which includes elements of description that are not present in other models, providing an indication as to which elements might have been missing from a given description. The proposed model is implemented in a web-based tool called Idea-Inspire 4.0, a brief introduction of which is also provided.
\end{abstract}

Keywords: analogical design; complex systems; function modelling; design tools; conceptual design; comprehension.

\section{INTRODUCTION}

Ideas generated for engineering design problems during the conceptual design phase anchor the development of detailed solutions in the following phases of design [1]. For generating ideas, engineering designers usually search for existing solutions that could readily offer (partial or complete) solution to the problem, without having the designer to reinvent the same idea [2]. There are web-based tools for searching solutions [3]-[6]. However, their search algorithms are not effective and result in a number of unspecific and irrelevant solutions.

A solution space, occasionally, might comprise out-of-thecontext, far-domain ideas (e.g. biological) that could provide 'novel' solutions to an engineering design problem [7]. However, it is usually a challenge for engineering designers to comprehend biological systems. When complex systems are provided as stimuli, designers often utilize entities and relationships that are present at their lower levels of abstraction, since the intertwined functioning of a complex system is often difficult to understand
[8]; this amounts to underutilization of stimuli and direct-transfer of analogies, which lead to designs of lower novelty [9], [10].

Systems are more complex when their components are larger in number, and function individually as well as interact with others in a larger number of ways [11], [12]. Representing (more) complex systems involves decomposition of their whole into smaller wholes, which on further decomposition, introduce more smaller wholes. At any step of the decomposition, there is a specific set of wholes constituting the system level, each performing an individual role and interacting with others; at any system level, an individual whole could be termed a sub-system.

The aim of this research is to represent the following: 1) subsystems at different system levels for the entire span of system decomposition; 2) a detailed description of each sub-system; and 3 ) interactions among these sub-systems. In this paper, we use a function model called SAPPhIRE as the basis and propose an approach to combine multiple instances of this model to capture those pieces of knowledge that together can be used represent the functioning of a complex system.

\section{BACKGROUND}

\subsection{FUNCTION, BEHAVIOUR, STATE}

Function-Behavior-State (including Structure) has been a longstanding approach to define the functioning of a system. The structure is used to explain the arrangement of physical elements, their properties and state variables at a given instance of time. This is also referred to as State in some parts of literature. Behaviour explains the sequence of states or processes that the system undergoes, in order to attain the desired state. The function could be the final state [13], interpretation of the final state [14], I/O relationship [15], or intended-effect [16] on the environment. These definitions have resulted in the development of SBF, FB-Structure, FB-State, FR, and many other representations that are reviewed in detail in [17], [18]. These models are also supported by tools such KRITIK, IDeAL, DANE, FBS modeller [2], [6], [19], [20].

\subsection{SYSTEM DECOMPOSITION METHODS}

The simplest way of decomposing a system is by developing a structural hierarchy of components and assigning a function to each of these [13], [21]. Alternatively, the overall function of the system could be represented as a sequence of processes, states, or sub-functions [22]-[24]. These sub-functions could be borrowed from a predefined set of vocabulary [24]. 


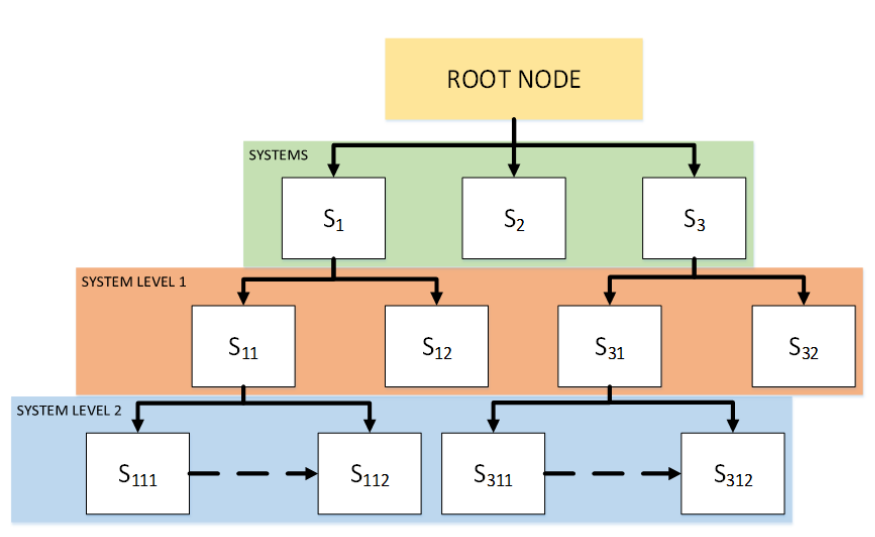

\begin{tabular}{|c|c|c|c|c|}
\hline No & Name & Parent & Link & Attributes \\
\hline 1 & ROOT & 0 & 0 & \\
\hline 2 & S1 & 1 & 0 & \\
\hline 3 & S2 & 1 & 0 & \\
\hline 4 & S3 & 1 & 0 & \\
\hline 5 & S11 & 2 & 0 & \\
\hline 6 & $\mathrm{~S} 12$ & 2 & 0 & \\
\hline 7 & S31 & 4 & 0 & \\
\hline 8 & $\mathrm{~S} 32$ & 4 & 0 & \\
\hline 9 & S111 & 5 & 0 & \\
\hline 10 & S112 & 5 & 9 & \\
\hline 11 & S311 & 7 & 0 & \\
\hline 12 & S312 & 7 & 11 & \\
\hline
\end{tabular}

Figure 1: a) System hierarchy built using structure or function decomposition b) Structuring the hierarchy into a database [11], [25]

The models based on FBS do not seem to adopt these system decomposition methods, and the following questions seem difficult to address in depth.

1. Can the overall function of a complex system be described using a single FBS model?

2. If not, which entity i.e., F, B, or S should drive the system decomposition?

3. Can every sub-system of a complex system be described using FBS model(s)?

4. In a subsystem, which entity $\mathrm{F}, \mathrm{B}$, or $\mathrm{S}$ is a subset or a portion of the FBS model of the overall system?

5. How is it possible to describe the connections, interactions, or relationships among sub-systems?

6. Which entity i.e., F, B, or $\mathrm{S}$ of a subsystem could be used to connect these to another subsystem? $\mathrm{F}_{1} \rightarrow \mathrm{S}_{2}$ or $\mathrm{F}_{1} \rightarrow \mathrm{B}_{2}$ or $\mathrm{F}_{1} \rightarrow \mathrm{F}_{2}$ or $\mathrm{B}_{1} \rightarrow \mathrm{B}_{2}$ ?

These issues arise because FBS literature was not initially introduced to represent complex systems. Adopting an FBS model for system decomposition methods poses a challenge, since the entities and relationships in an FBS model do not seem to support these. Hence, as an alternative to FBS model, we propose SAPPhIRE model, a more recent model that had been developed as an integration of FBS literature and several other function theories.

\section{A DESCRIPTIVE MODEL FOR COMPLEX SYSTEMS 3.1. SYSTEM DECOMPOSITION}

Let us consider three systems $-S_{1}, S_{2}$, and $S_{3}$, as shown in Figure 1a. These systems can be represented in a database, which is denoted by a 'root node' on the top of the hierarchy in Figure 1a. Usually, databases comprise tables in which information pertinent to these models are stored as text, numbers, characters etc. In order to translate this model into a database, it could be structured into a tabular format shown in Figure 1b using a set of 'attributes' derived from their function models.

If these systems are complex and require an in-depth explanation, each system must be decomposed into subsystems such as $S_{11}, S_{12}, S_{31}$ and $S_{32}$. If these sub-systems were to be included in the table, we could include a separate column called 'parent' where Parent $\left[S_{11}\right]=2$ signifies that super system $S_{1}$ is present at the $2^{\text {nd }}$ serial number. This way, it is possible to structure that hierarchical relationship. These subsystems, too, can be structured using 'attributes' similar to their super-systems $-S_{1}, S_{2}$, and $S_{3}$; the uniformity in describing systems and subsystems is usually not taken into account in the FBS literature [14], [22], [24].

If we decompose $S_{11}$ and $S_{31}$ further, another system level is introduced with new sub-systems such as $\mathrm{S}_{111}, \mathrm{~S}_{112}, \mathrm{~S}_{311}$, and $\mathrm{S}_{312}$. It is possible that these sub-systems could be causally connected i.e., the output of one becomes the input of the other, like the dashed link shown between $S_{111} \rightarrow S_{112}$ and $S_{311} \rightarrow S_{312}$. These links could also be structured into a tabular form by using a 'link' column, where Link $\left[S_{112}\right]=9$ signifies that sub-system $S_{112}$ is on the tail end of a link originating from a system with serial number 9 , which is $S_{111}$.

The system decomposition that we have shown does not only consider detailing a system but also developing a database structure that could support the decomposition, which is important for developing search tools in order to support, e.g. analogical design. From this decomposition, we could extract the following points.

1. A system could be decomposed to an arbitrary level of detail, but each sub-system introduced must be treated indifferently compared to its super-systems; a set of attributes that define the overall would also uniformly represent each (sub-) system.

2. The set of attributes that we choose for each (sub-) system must explain the hierarchical and causal relationships without compromising the information on how the connectivity was established.

Models based on FBS terminology do not directly fit into the above two points. As mentioned earlier in Section 2.2, this is mainly because the entities and relationships in FBS model do not seem to support system-decomposition methods. In the following section - 3.2, we adopt the above system decomposition approach and integrate with SAPPhIRE model. 


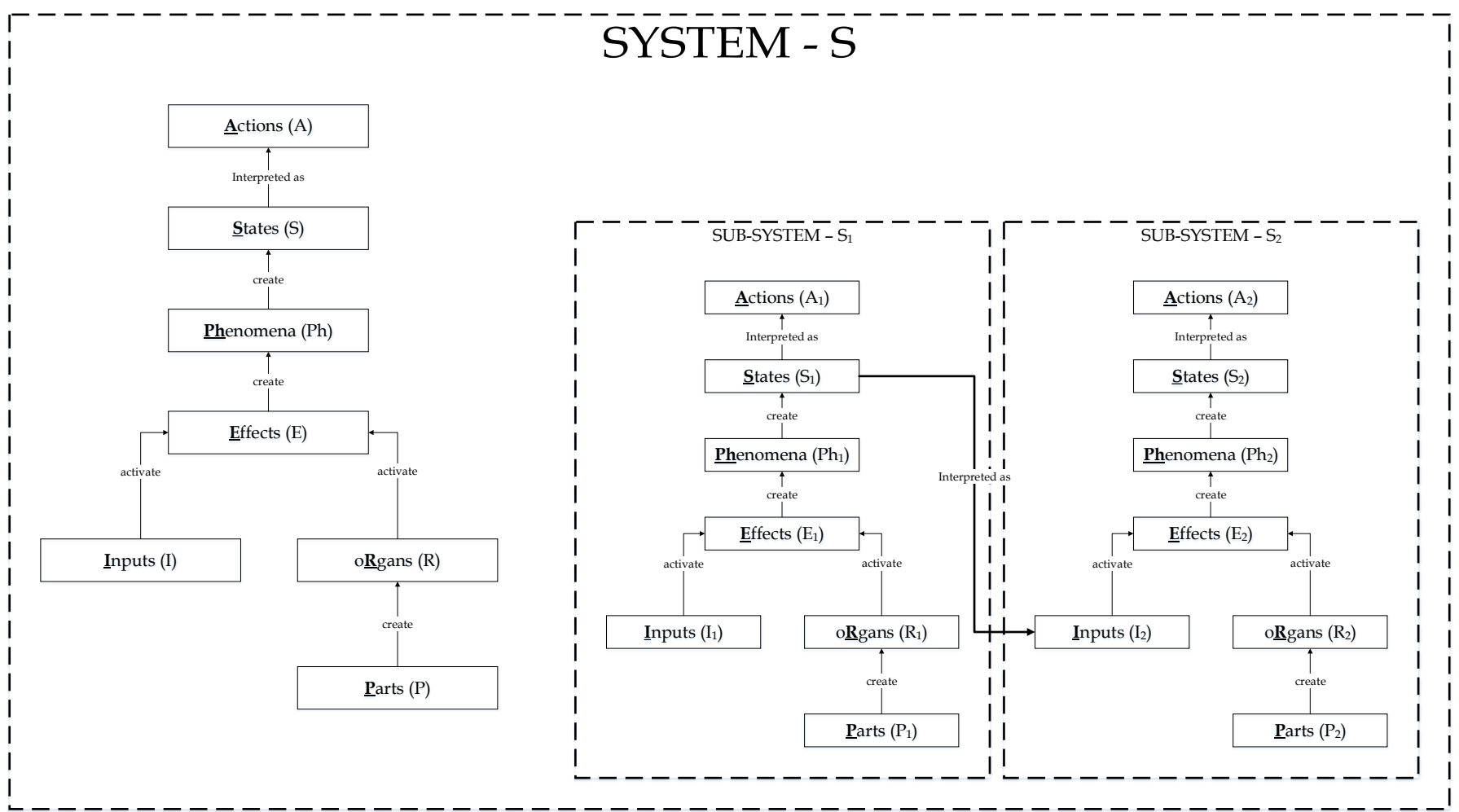

Figure 2: Multiple-instance SAPPhIRE model

\subsection{MULTIPLE-INSTANCE SAPPHIRE MODEL}

SAPPhIRE stands for $\underline{\text { States, }}$ Actions, $\underline{\text { Parts, }}$ Phenomena, Inputs, o $\underline{\mathbf{R} g a n s}$, and $\underline{\mathbf{E}}$ ffects. These entities explain the functioning of a system at different levels of abstraction. While Srinivasan and Chakrabarti [26] provide detailed definitions of these entities, a brief description is provided below (Figure 2).

Parts are components and interfaces that constitute the system. Derived from the $\underline{\mathbf{P}}$ arts are the o$\underline{\mathbf{R} g a n s}$ that are properties and conditions required for the system to function. Input is a physical quantity in the form of material, energy, or information that enters the system boundary and instigates functioning of the system. Phenomena are the physical processes governed by

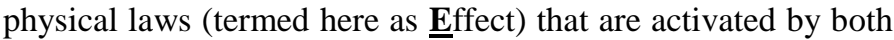
Input and oㅁgans. The $\underline{\mathbf{P h}}$ enomena are the response for the exchange of materials, energy etc., between an entity and its surrounding, leading to change in state variables or properties of the entity, which is denoted by the construct $-\underline{S}$ tates. Finally, the change of $\underline{\mathbf{S}}$ tates is interpreted as $\underline{\mathbf{A}} \mathbf{c t i o n s}$, which is a higher level

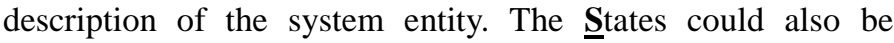
interpreted as Inputs.

The above entities of SAPPhIRE were used in earlier work to structure information about over 1200 biological and engineered systems into a searchable knowledge base, which was implemented in a standalone tool called Idea-Inspire [27]. These systems were treated as a single instance of the SAPPhIRE model and modelled using one SAPPhIRE model each. A number of biological systems such as 'albatross' (flying bird) and 'mudskipper' (rock climbing fish), it was found, could be used to provide stimuli for developing useful solutions to engineering design problems. However, due to the complexity of these systems, it was not possible to represent using these single SAPPhIRE instances, which limited their utility in the analogical design process. Therefore, in this research, we propose to decompose such systems and represent them in detail using multiple instances of SAPPhIRE, which we label as multipleinstance SAPPhIRE model (Figure 2).

\subsubsection{HIERARCHICAL RELATIONSHIP}

In Figure 2, the overall system boundary, which is labelled as 'SYSTEM - S' denotes the main system, whose SAPPhIRE model is shown on the left-hand side. If this system is decomposed w.r.t. components (e.g. a motor decomposed into $\rightarrow$ switch, rotor, stator), it gives rise to sub-systems $S_{1}, S_{2}$ etc., that are shown are separately within their system boundaries. This

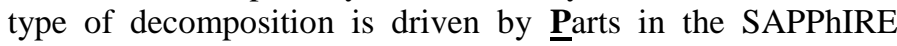
model. Then, we would assign Actions (roles for each component) and complete the explanation.

If the decomposition is carried out w.r.t. process (e.g. Otto cycle decomposed into the following sub-systems that function in sequence: intake stroke $\rightarrow$ compression stroke $\rightarrow$ power stroke $\rightarrow$ exhaust stroke), new system boundaries need not be formed, but the sub-systems must be separated by time i.e., they must function in sequence.

Using Parts based, Phenomena based, or combined decomposition, a hierarchical relationship is established. In addition, each sub-system $\left(S_{1}\right.$ or $\left.S_{2}\right)$ is also capable of being represented as SAPPhIRE model, similar to how the overall system $\mathrm{S}$ is represented. 


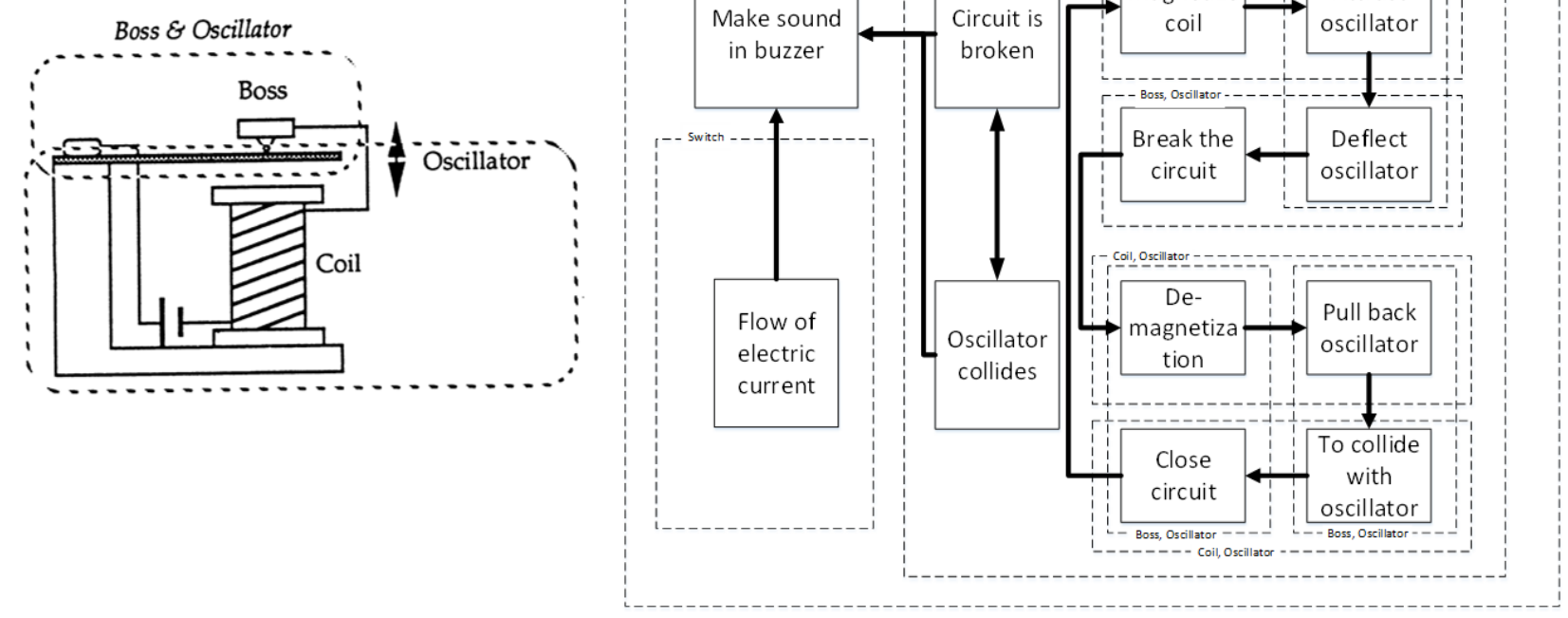

Figure 3: a) A schematic diagram and b) multiple-instance SAPPhIRE model of an electric buzzer

\subsubsection{CAUSAL RELATIONSHIP}

In Figure 2, there is a link from the States of $S_{1}$ to Inputs of $S_{2}$ indicating that there is a causal relationship between these. The States $\stackrel{\text { interpreted as }}{\longrightarrow}$ Inputs was earlier proposed by Chakrabarti et al. [27], which is used in this paper to establish a causal

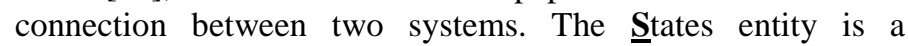

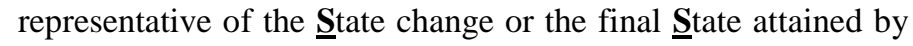
the system, which in a sense denotes the output.

First, in order to establish this connection, $S_{1}$ and $S_{2}$ must be separated by time. If two sub-systems function simultaneously, the input of one sub-system cannot depend on the output of the other. Second, this link cannot be established if $\underline{\text { States }} \rightarrow \underline{\text { Inputs }}$ pair is not obvious. For instance, intake stroke cannot be causally connected to power stroke because the Inputs for power stroke depend only upon the $\underline{\mathbf{S}}$ tates described by compression stroke. If such obviousness cannot be seen, the process must be decomposed further.

This causal relationship along with the aforementioned hierarchical relationship promises a powerful combination for connecting sub-systems in a complex system; this is demonstrated using an example in the following section.

\section{EXAMPLE}

We use an electric buzzer (Figure 3a) as an example to illustrate the potential of multiple-instance SAPPhIRE model. In Figure $3 \mathrm{~b}$, the complete multiple-instance SAPPhIRE model for electric buzzer is shown. The ultimate Actions, 'Make sound using buzzer' is achieved using three sub-functions, 'Flow of electric current', 'Circuit is broken', and 'Oscillator collides' that occur in different inner system boundaries. These boundaries are further explored by decomposing their sub-systems into four sub-sub-systems each.
The overall function of the electric buzzer is shown in Figure 4.

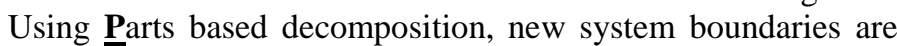
formed, one for the switch and another for the rest. The model for switch explains as to how electric current is produced and enters the circuit as energy Input. The coil gets magnetized due to current flow and attracts the oscillator. Hence, the oscillator detaches itself from the contact with the boss, which results in the broken circuit.

The displacement of the oscillator (ㅁtes) is interpreted as Input for another subsystem, which explains as to how oscillator uses spring force to hit the boss (슬ions). The contact with the boss (States) completes the circuit, which is interpreted as the flow of electric current (Inputs) for the previous subsystem. Therefore, these two subsystems, 'circuit is broken' and 'oscillator collides', function alternatively as shown in Figure 4 and $3 \mathrm{~b}$.

\section{SUMMARY, DISCUSSION, AND CONCLUSIONS 5.1. COMPLETENESS OF THE PROPOSED MODEL}

1. In the SAPPhIRE model shown in Section 3.2, the entities themselves are used for decomposing the system and therefore the sub-systems generated could replicate the overall SAPPhIRE model, also shown using electric buzzer example in Figure 4. In addition, the entities of SAPPhIRE model provide an in-depth explanation of each sub-system, also indicating the absence of information wherever applicable.

2. In the SAPPhIRE model, hierarchical relationships are clearly established using system boundaries; when Parts based decomposition is carried out, a separate boundary is created for the switch in Figure 4. In the case of processbased decomposition, Phenomena are divided into two subsystems of electric buzzer (Figure 4). These sub-systems are also causally connected using $\underline{\text { States }} \rightarrow$ Inputs link. 


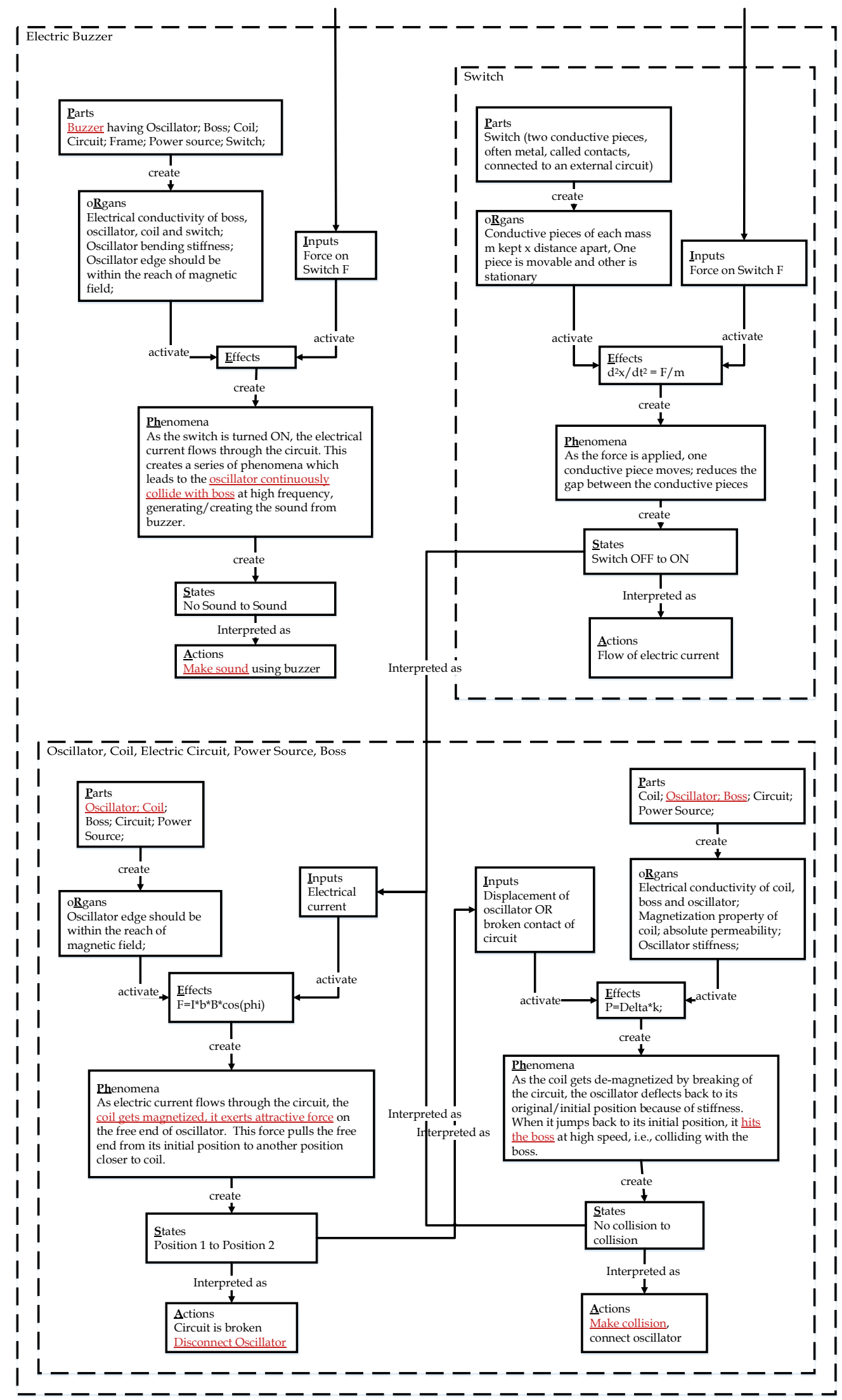

Figure 4: The expanded multiple-instance SAPPhIRE model of an electric buzzer 


\section{climbing of mudskipper}

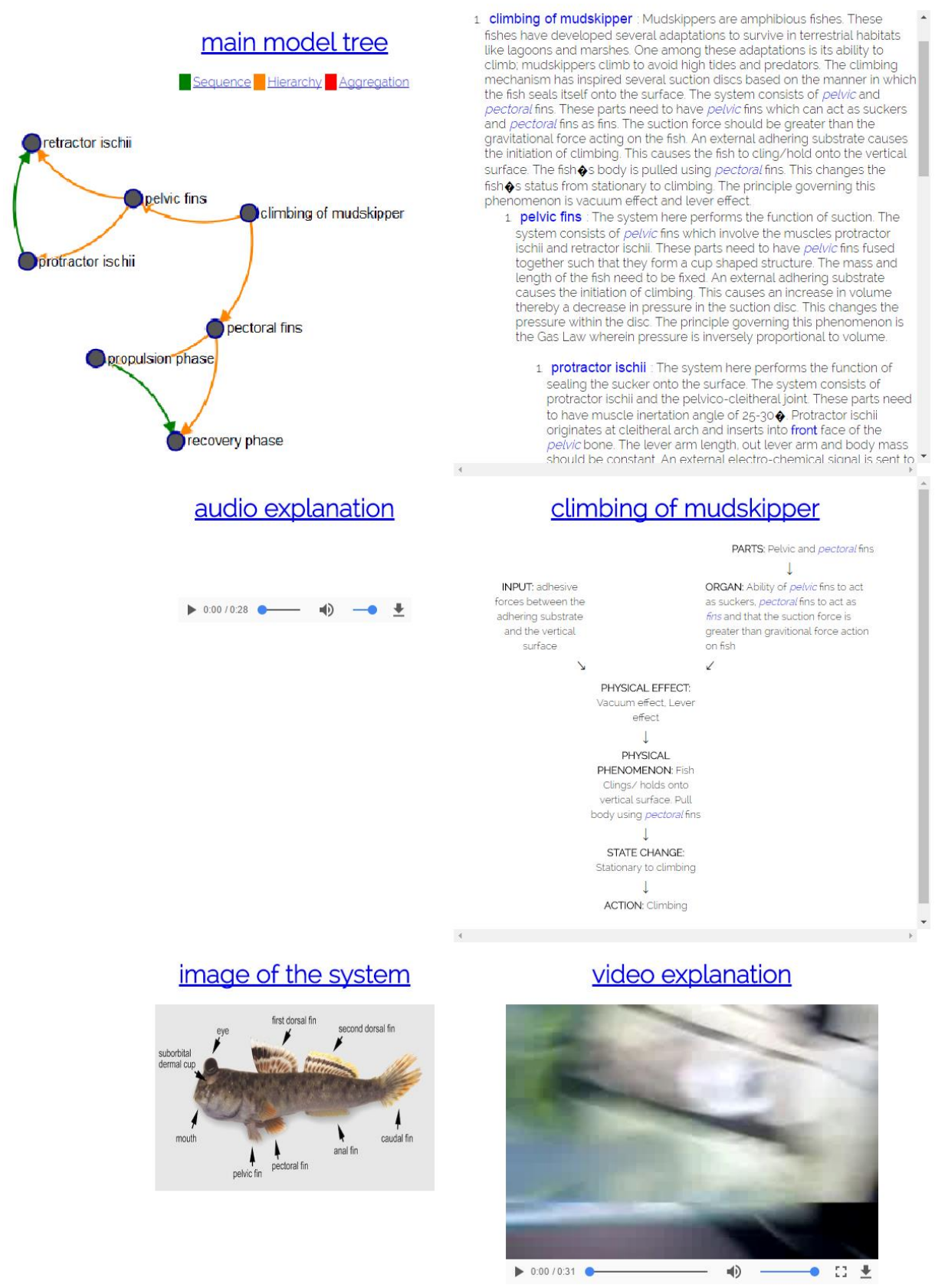

Figure 5: Idea-Inspire 4.0: a multi-modal representation 


\subsection{IDEA-INSPIRE 4.0}

We have developed an upgraded version of Idea-Inspire [27] to support multiple instance SAPPhIRE models and a new database structure for system decomposition shown in Figure 1b. The newer version -4.0 is significantly improved with new features, see Chakrabarti et al. [28]. In Figure 5, a screenshot of IdeaInspire 4.0, depicting a system 'climbing of a mudskipper', is provided.

The graph in the top-left portion of Figure 5 is the multipleinstance model for mudskipper, in which both hierarchical and causal relationships defined in Section 3.2 are used. This biological example, along with 142 other systems in the IdeaInspire 4.0 database, demonstrates the capability of the multipleinstance SAPPhIRE model for modelling complex systems, from both artificial and biological domains. The other elements of representation include a textual explanation, audio, images, and videos. In this paper, we have only covered the multiple-instance model.

According to [29], general linguistic representations of analogous systems are helpful in aiding idea-generation. Based on this observation, we are currently in the process of modelling textual explanations (top-right) using Controlled Natural Language (CNL) templates and developing translation techniques for these. Images and videos included in the representation are a result of [30], which says that the number of ideas generated in a design session using stimuli could be improved using digital support such as audio, video, and images. Currently, every image and video in Idea-Inspire 4.0 are available in open-source.

\subsection{COMPARISON WITH THE FB-STATE MODEL}

In this section, we compare the multiple-instance SAPPhIRE model with the FB-State model [14] that was illustrated using the electric buzzer. The FBS model of electric buzzer example is available in [14, p. 187]; our model is shown in Figures $3 \& 4$, for the following points of comparison. Also in Figure 4, we have underlined the information that was present in Umeda et al. [14, p. 187].

1. As a general observation, $\underline{\mathbf{P}}$ arts and o$g a n s$, in comparison to components, attributes and relations in the FBS model provide a clear structural context of the system, mainly due to the clearly-stated definitions and system-boundary.

2. Effects such as the magnetic force (F) is activated by oRgans and Inputs like electric current (I), the magnetic field produced in the coil (B), the width (b), and the angle of orientation (phi). The FBS model only states a view (e.g. mechanics) instead of specific relationships among these.

3. Phenomena explain as to how a scientific relation (Effects) activated by Inputs gives to an exchange lead to a change in

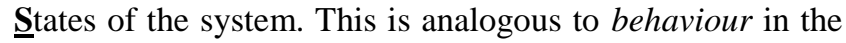
FBS model; however, FBS model does not explain as to what instigates the behaviour unlike the use of Inputs.

4. The information provided in the FBS model, if cast in SAPPhIRE model terms, would provide an organized explanation, that supports the identification of aspects of the system that are not explained. For instance, in the FBS model, "oscillator is pulled by magnetic force" does not mention that electric current (Inputs) was responsible for the magnetic force, and FBS model does not have a provision to point out this lack of information.

5. We have decomposed the system using both Parts and Phenomena based techniques. In the FBS model, the functional hierarchy is created based on different views such as mechanics, magnetism, etc. Identification of views could be subjective and the number of views could be arbitrary.

6. In the FBS model, the causal connection between two subfunctions, 'oscillator collides' and 'circuit is broken' is not explained clearly because the relationship itself is not defined. In SAPPhIRE model, we use the $\underline{\text { States }} \rightarrow \underline{\text { Inputs }}$ relationship to make a causal connection.

7. FBS model does not include information of what triggers the function from outside the system boundary. This is explained using Inputs in the SAPPhIRE model, which for instance led to the construction of a new model for 'switch' to explain as to how electric current flows into the circuit. In addition, the presence of Inputs indicated the inadequacy of information in this case.

As mentioned earlier in Section 2.2, the above-stated deficits of FBS model because F-B-S literature does not stem from the idea that systems could be complex and the sub-systems of these could interact in multiple ways. For instance, Goel et al. [6] developed a tool called DANE to support analogical design. In this paper, Goel et al. [6] claim to have used SBF model for describing complex systems. However, in their tool, one or more systems are connected to a system, which is supposedly the super-system of these. By doing so, Goel et al. [6, pp. 884, 887] seem to claim that DANE enables understanding of complex analogues through decomposition. However, there is absolutely no information on how a system is decomposed, i.e., which entity $\mathrm{S}, \mathrm{B}$, or $\mathrm{F}$ is decomposed, how the decomposition is carried out, or if at all decomposition is done. Next, there is no information on systems at the same level are connected i.e., how S, B, and F entities interact with those of other systems. This caveat has been addressed in our model (See Figures 2 and 4).

Adding to these, we argue, the modes of description used in DANE appear less comprehensive, although it is claimed in the paper that features of DANE were developed out of a thorough background research on cognition [6, p. 296]. Our argument is based on the observation that a user must switch between several windows to gather information about one system. Idea-Inspire 4.0 , on the other hand, provides an organised explanation of a sub-system on a single page, whose template is uniformly applied for other sub-systems as well.

Moreover, under behavioural description, Goel et al. [6, p. 892] provide, merely a large amount of text supported by some images, which is no different from AskNature, Wikipedia etc. Due to the haphazard manner in which Goel et al. [6, pp. 889895] describe a system shows that the role of SBF model in the tool is trivial. Textual-description is a part of the explanation in 
Idea-Inspire 4.0; however, it follows a particular template of fixed length that moulds only the information present in SAPPhIRE model, which is also responsible for explaining the connection between two sub-systems in the multiple-instance model (see Figure 5, top-right). Adding to these, SAPPhIRE entities form the basis by which Idea-Inspire 4.0 database is structured (See Figure 1b) and search algorithm is created. Therefore, unlike SBF model in Goel et al. [6], SAPPhIRE model orchestrates the entire features of Idea-Inspire 4.0.

The above issues of DANE were mainly due to lack of training to develop SBF models. DANE's knowledge base was developed in an undergraduate classroom, where students might have, typically no experience on complex biological systems, which is why, according to Goel et al. [6, p. 891], they were asked to understand several of these systems from other knowledge bases. In addition, Wiltgen et al. [31] report that students were less interested in encoding the open source knowledge into SBF models, which were to be populated into DANE.

An extensive discussion was carried out on DANE here, since it is the most recently implemented tool on $\mathrm{F}, \mathrm{B}$, and $\mathrm{S}$ way of describing systems. However, in spite of the claims of Goel et al. [6], the tool stands merely as a knowledge base in which, SBF seems to play a less significant role in system decomposition and explaining individual systems. Therefore, we anticipate, there are fundamental issues with $\mathrm{F}, \mathrm{B}$, and $\mathrm{S}$ terminologies that pose a challenge to answer the aforementioned six questions (See Section 2.2) w.r.t., system decomposition. In order to exemplify these, it was necessary to visit the earliest work on F, B, and S [14] as done in this section. The outcome of comparing SAPPhIRE and FBS leaves us with a conjecture that SAPPhIRE is more comprehensive and flexible for systems with varying levels of complexity.

\subsection{FUTURE DIRECTIONS}

In view of Idea-Inspire 4.0 , it is desirable that systems are explained in future using more realistic representations such as Virtual Reality (VR) videos and holograms. However, currently, these are not readily accessible and affordable. The current version of Idea-Inspire 4.0 supports only text-based search; future work should include adopting Digital Image Processing (DIP) techniques to engage in image-based retrieval. A substantial human effort is involved in developing large knowledge bases such as Idea-Inspire 4.0, which needs to be automated in future, whose initial success is reported in Keshwani and Chakrabarti [32].

\section{REFERENCES}

[1] K. T. Ulrich, Product design and development. Tata McGraw-Hill Education, 2003.

[2] S. Bhatta, A. Goel, and S. Prabhakar, "Innovation in analogical design: A model-based approach," in Artificial Intelligence in Design'94, 1994, pp. 57-74.

[3] Encyclopedia. http://www.encyclopedia.com/, 2017.

[4] Wikipedia. https://en.wikipedia.org/wiki/Main_Page, 2017.
[5] J.-M. Deldin and M. Schuknecht, "The AskNature database: enabling solutions in biomimetic design," in Biologically inspired design, Springer, 2014, pp. 17-27.

[6] A. K. Goel, S. Vattam, B. Wiltgen, and M. Helms, "Cognitive, collaborative, conceptual and creative-four characteristics of the next generation of knowledge-based CAD systems: a study in biologically inspired design," Computer-Aided Design, vol. 44, no. 10, pp. 879-900, 2012.

[7] K. Fu, J. Chan, J. Cagan, K. Kotovsky, C. Schunn, and K. Wood, "The meaning of 'near' and 'far': the impact of structuring design databases and the effect of distance of analogy on design output," Journal of Mechanical Design, vol. 135, no. 2, p. 021007, 2013.

[8] S. Keshwani and A. Chakrabarti, "Influence of analogical domains and abstraction levels on novelty of designs," in 3rd international conference on design creativity, Bangalore, 2015, pp. 062-069.

[9] S. Keshwani and A. Chakrabarti, "Influence of analogical domains and comprehensiveness in explanation of analogy on the novelty of designs," Springer, no. Research in Engineering Design, pp. 1-30, 2016.

[10] S. S. Vattam, M. E. Helms, and A. K. Goel, "A content account of creative analogies in biologically inspired design," Artificial Intelligence for Engineering Design, Analysis and Manufacturing, vol. 24, no. 04, pp. 467-481, 2010.

[11] L. Siddharth and P. Sarkar, "A Multiple-Domain Matrix Support to Capture Rationale for Engineering Design Changes," Journal of Computing and Information Science in Engineering, vol. 18, no. 2, pp. 021014-021014-11, Apr. 2018.

[12] L. Siddharth and P. Sarkar, "A Methodology for Predicting the Effect of Engineering Design Changes," Procedia CIRP, Elsevier, vol. 60, pp. 452-457, 2017.

[13] A. K. Goel and B. Chandrasekaran, "Functional Representation of Designs and Redesign Problem Solving.," in IJCAI, 1989, pp. 1388-1394.

[14] Y. Umeda, H. Takeda, T. Tomiyama, and H. Yoshikawa, "Function, behaviour, and structure," Applications of artificial intelligence in engineering $V$, vol. 1, pp. 177193, 1990.

[15] G. Pahl and W. Beitz, Engineering Design: A Systematic Approach, Springer. Springer-verlag London, 2007.

[16] B. Chandrasekaran and J. R. Josephson, "Function in device representation," Engineering with computers, vol. 16, no. 3, pp. 162-177, 2000.

[17] A. Chakrabarti, V. Srinivasan, B. Ranjan, and U. Lindemann, "A case for multiple views of function in design based on a common definition," AI EDAM, vol. 27, no. 3, pp. 271-279, 2013.

[18] M. S. Erden, H. Komoto, T. J. van Beek, V. D’Amelio, E. Echavarria, and T. Tomiyama, "A review of function modeling: approaches and applications," Artificial Intelligence for Engineering Design, Analysis and Manufacturing, vol. 22, no. 02, pp. 147-169, 2008. 
[19] A. Goel, S. Bhatta, and E. Stroulia, "Kritik: An early casebased design system," Issues and applications of casebased reasoning in design, vol. 1997, pp. 87-132, 1997.

[20] Y. Umeda, M. Ishii, M. Yoshioka, Y. Shimomura, and T. Tomiyama, "Supporting conceptual design based on the function-behavior-state modeler," AIEDAM, vol. 10, no. 4, pp. 275-288, 1996.

[21] K. Ulrich, "The role of product architecture in the manufacturing firm," Research policy, vol. 24, no. 3, pp. 419-440, 1995.

[22] B. Chandrasekaran, A. K. Goel, and Y. Iwasaki, "Functional representation as design rationale," Computer, vol. 26, no. 1, pp. 48-56, 1993.

[23] V. Sembugamoorthy and B. Chandrasekaran, Functional representation of devices and compilation of diagnostic problem solving systems. 1984.

[24] R. B. Stone and K. L. Wood, "Development of a functional basis for design," Journal of Mechanical design, vol. 122, no. 4, pp. 359-370, 2000.

[25] L. Siddharth, P. Sarkar, and A. Chakrabarti, "Modelling and structuring the knowledge of failures using Design Structure Matrix (DSM) for reuse during product lifecycle," presented at the 6th International Conference on Product Lifecycle Modeling, Simulation and Synthesis PLMSS 2017, DIAT, Pune, India, 2017, pp. 104-115.

[26] V. Srinivasan and A. Chakrabarti, "SAPPhIRE-an approach to analysis and synthesis," in DS 58-2: Proceedings of ICED 09, the 17th International Conference on Engineering Design, Vol. 2, Design Theory and Research Methodology, Palo Alto, CA, USA, 24.27.08. 2009, 2009.

[27] A. Chakrabarti, P. Sarkar, B. Leelavathamma, and B. Nataraju, "A functional representation for aiding biomimetic and artificial inspiration of new ideas," AIE EDAM, vol. 19, no. 02, pp. 113-132, 2005.

[28] A. Chakrabarti, L. Siddharth, M. Dinakar, M. Panda, N. Palegar, and S. Keshwani, "Idea-Inspire 3.0 - A Tool for Analogical Design," presented at the International Conference on Research into Design (ICoRD'17), 2017, vol. 2, p. 10.

[29] J. Linsey, K. Wood, and A. Markman, "Modality and representation in analogy," Artificial Intelligence for Engineering Design, Analysis and Manufacturing, vol. 22, no. 02, pp. 85-100, 2008.

[30] P. Sarkar and A. Chakrabarti, "The effect of representation of triggers on design outcomes," AIEDAM (Artificial Intelligence for Engineering Design, Analysis and Manufacturing), vol. 22, no. 2, p. 101, 2008.

[31] B. Wiltgen, S. Vattam, M. Helms, A. K. Goel, and J. Yen, "Learning functional models of biological systems for biologically inspired design," in Advanced Learning Technologies (ICALT), 2011 11th IEEE International Conference on, 2011, pp. 355-357.

[32] S. Keshwani and A. Chakrabarti, "Towards Automatic Classification of Description of Analogies into SAPPhIRE Constructs," in International Conference on Research into Design, 2017, pp. 643-655. 